\title{
El capital intelectual en el reporte integrado: un estudio exploratorio en el mercado de capitales argentino a partir del análisis de datos textuales
}

\section{Intellectual capital in the integrated reporting: an exploratory study in the Argentinian stock market based on textual analysis}

\author{
Cecilia Ficco ${ }^{* 1}$ \\ Universidad Nacional de Río Cuarto \\ cficco@fce.unrc.edu.ar \\ Gabriela García ${ }^{1}$ \\ Universidad Nacional de Río Cuarto \\ ggarcia@fce.unrc.edu.ar \\ Gustavo Sader ${ }^{1}$ \\ Universidad Nacional de Río Cuarto \\ gsader@eco.unrc.edu.ar \\ Jonathan Luna Valenzuela' \\ Universidad Nacional de Río Cuarto \\ jvalenzuela@fce.unrc.edu.ar
}

\author{
Autor corresponsal. \\ 1 Universidad Nacional de Río Cuarto, \\ Facultad de Ciencias Económicas, \\ Ruta 36 km. 601, Río Cuarto, Córdoba \\ ARGENTINA.
}

\section{Resumen}

Propósito: Este artículo pretende analizar la divulgación de información sobre capital intelectual (CI) en el reporte integrado (RI), a los efectos de mostrar la complementariedad entre ambos enfoques.

Diseño/metodología: La investigación de carácter exploratorio combina una revisión bibliográfica con un estudio empírico basado en el análisis de datos textuales.

Resultados: Las evidencias obtenidas indican una asociación entre el marco conceptual del Cl y el marco de referencia del RI. Asimismo, se identificaron empíricamente elementos asociados al $\mathrm{Cl}$ y a sus componentes en los RI Implicaciones: LoS RI constituyen un importante desarrollo en las prácticas de divulgación de información sobre $\mathrm{Cl}$, reivindicado su relevancia en los procesos de creación de valor.

Limitaciones: El número de empresas y el período analizados son limitados, dado que el RI es una práctica incipiente en Argentina.

Originalidad/valor: El estudio contribuye al conocimiento de las prácticas de divulgación sobre $\mathrm{Cl}$ en el RI en el contexto latinoamericano, utilizando una metodología novedosa.
INFORMACIÓN ARTÍCULO

Recibido: 5 de Enero 2021

Aceptado: 29 de Marzo 2021
Palabras Claves:

Capital intelectual

Reporte integrado

Análisis textual 


\begin{abstract}
Purpose: This paper aims to assess intellectual capital (IC) disclosures in the integrated report (IR), in order to determine the complementarity between these frameworks.

Design/methodology: An exploratory research was conducted by integrating a literature review with a non-experimental empirical study based on textual analysis.

Findings: Results indicate an association between the theoretical framework for IC and the International IR Framework. Moreover, IC disclosures were identified empirically in the IR.

Practical implications: IR is an important development in the corporate IC disclosure practices, asserting their relevance to organizational value creation.

Research limitations: Since IR is an emerging practice in Argentina, the number of companies under study was limited.

Originality/value: The study provides insights into IC disclosure practices in the IR in the Latin American context by applying an innovative methodological approach.
\end{abstract}

ARTICLE INFO

Received: 5 January 2021

Accepted: 29 March 2021

\section{Keywords:}

Intellectual capital Integrated report

Textual analysis

\section{INTRODUCCIÓN}

La creación de valor por parte de las organizaciones se realiza mediante una amplia gama de actividades, interacciones y relaciones, las cuales resultan significativas para distintos grupos de interés. Los estados financieros (EF), con sus notas y anexos, constituyen una de las fuentes utilizadas por dichos grupos para conocer la situación y evolución de la empresa y, a través de ellos, se revela información que muestra diversos aspectos de interés sobre el proceso de creación de valor. Entre esa información se destaca la referida al $\mathrm{Cl}$, en virtud de que el conocimiento, componente fundamental de dicho capital, parece erigirse como un recurso clave en la generación de riqueza organizacional en el actual contexto (Ficco, 2020a; Azofra Palenzuela, Ochoa Hernández, Prieto Moreno y Santidrián Arroyo, 2017). En este sentido, el $\mathrm{Cl}$ refiere al conjunto de elementos intangibles (recursos, capacidades y actividades) relacionados entre sí, en el que destaca el conocimiento disponible, tanto a nivel individual como organizativo, que permite a la empresa funcionar en combinación con los demás recursos inherentes a la misma, siendo fuente de ventaja competitiva y de creación de valor (Edvinsson y Malone, 1997; Stewart, 1998; Bueno, Salmador y Merino, 2008; Ficco, 2020b).

Sin embargo, dado que es muy limitada la información sobre $\mathrm{Cl}$ que se incorpora dentro de la obligatoria y que forma parte de los EF, porque involucra solo la referida a los intangibles que pueden reconocerse como activos, la alternativa más usada por las empresas para dar a conocer su $\mathrm{Cl}$, es la de divulgar esta información de forma totalmente voluntaria, usando para ello diversos formatos y canales (Castilla y Ruiz, 2017; Ficco y Sader, 2020). Uno de ellos está dado por la información complementaria a los EF, incorporada en las notas a los EF y en la memoria, dentro de la cual se incluye, principalmente, información no financiera de tipo cualitativo.

Otro de los medios que permite a las organizaciones revelar información que puede aportar a mejorar la concepción de valor y su creación a través del tiempo es el RI, cuya presentación tiene carácter voluntario en Argentina. El RI, propuesto por el International Integrated Reporting Council (IIRC), tiene como propósito principal "explicar a los proveedores de capital financiero cómo una organización crea valor a lo largo del tiempo" (IIRC, 2013, p. 8), brindando información acerca de "los recursos y las relaciones utilizados y afectados por la organización [esto es, sus capitales]" (IIRC, 2013, p. 11) y explicando, además, cómo “interactúa con el entorno externo y con los capitales para crear valor en el corto, medio y largo plazo" (IIRC, 2013, p. 11).

Bajo el enfoque descrito, la estrategia de revelación incluye distintas categorías de capitales que "son inventarios de valor que se incrementan, disminuyen y transforman a través de las actividades y las salidas de la organización" (IIRC, 2013, p. 12). Esos capitales refieren tanto a bienes físicos como a recursos y actividades intangibles 
y se agrupan "en seis categorías de capitales: financiero, industrial, intelectual, humano, social y relacional, y natural" (IIRC, 2013, p. 5).

Se aprecia, de este modo, un importante problema en lo relativo a la información sobre el $\mathrm{Cl}$ de las empresas, que da lugar a significativas deficiencias informativas con relación al conjunto de elementos intangibles que resultan clave para la creación de valor. Este problema se configura ante la falta de un marco general para la elaboración de un informe específico de $\mathrm{Cl}$, que deja librada a la voluntad de las empresas la decisión de revelar información sobre sus distintos componentes, tanto en los aspectos de cantidad y calidad de información a revelar, como de medio o canal a emplear para la divulgación.

La problemática antes planteada, ha contribuido al desarrollo de una línea de investigación enfocada en el análisis de la divulgación voluntaria de $\mathrm{Cl}$ que realizan las empresas. En esta línea se ubican los trabajos centrados en describir las prácticas de divulgación de $\mathrm{Cl}$ adoptadas por las empresas, la mayoría de los cuales se enfocan, principalmente, en la cantidad de información revelada (De Silva, Stratford y Clark, 2014; Al-Hajaya, Altarawneh y Altarawneh, 2019), concluyendo sobre la tendencia creciente a divulgar información voluntaria respecto al $\mathrm{Cl}$.

En lo que respecta a los medios o canales de comunicación utilizados, los EF constituyen el documento más revisado al analizar la divulgación de Cl (Sihotang y Sanjaya, 2014; Tejedo, 2016), lo que ha dado lugar, tal como señalan Castilla y Ruiz (2017), a una de las principales críticas que ha recibido la literatura existente en esta línea. En este sentido, Dumay y Cai (2014), han enfatizado en la necesidad de estudiar la divulgación de información sobre Cl en el contexto del RI, a efecto de que las investigaciones contribuyan a desarrollar nuevos conocimientos sobre el Cl. Sin embargo, los estudios que han considerado al RI como fuente de divulgación de Cl son comparativamente más escasos, destacándose los de Melloni (2015), Beretta, Demartini y Trucco (2019) y Salvi, Vitolla, Giakoumelou, Raimo y Rubino (2020). En lo que respecta, en particular, al ámbito latinoamericano, ni siquiera se ha generalizado aún la adopción del RI como práctica corporativa de divulgación de información (Rivera Arrubla, Zorio Grima y García Benau, 2016), situación que queda claramente evidenciada en el estudio de Macias y Farfan Lievano (2017), sobre el proceso de implementación del RI en Colombia. Por último, es necesario des- tacar que gran parte de los estudios antes mencionados utiliza el análisis de contenido como estrategia metodológica para estudiar la divulgación voluntaria de Cl, lo que también ha sido objeto de diversas críticas, tal como queda plasmado en el trabajo de Dumay y Cai (2014).

De esta manera, en el marco del problema planteado y teniendo en cuenta las limitaciones señaladas por la literatura previa, el presente trabajo se propone analizar la presentación de información sobre el Cl de las empresas, compatibilizando la concepción clásica que incluye tres dimensiones para el mismo (humana, relacional y estructural), con el soporte conceptual del RI del IIRC, como estrategia organizacional para comunicar información de carácter financiero y no financiero, que permite mostrar cómo se desarrolla el proceso de creación de valor en el tiempo. Concretamente, el objetivo fundamental de este trabajo es analizar la divulgación de información sobre Cl en el RI, con el propósito de mostrar la complementariedad de ambos enfoques.

Para dar cumplimiento al objetivo planteado, se realiza un estudio exploratorio que combina una revisión bibliográfica destinada a mostrar la complementariedad de ambas concepciones a nivel teórico, con la aplicación de un diseño no experimental para la realización de un estudio empírico que permite analizar, en las prácticas de divulgación de información, aquella que refiere al Cl y que se canaliza a través del RI. El estudio empírico se sustenta en el análisis de datos textuales y se contextualiza en el mercado de capitales argentino. Específicamente, se trabaja con las empresas listadas en dicho mercado que han publicado su RI elaborado a partir del marco de referencia del IIRC, siendo el período de análisis 2018-2019.

De este modo, este artículo contribuye a aumentar el conocimiento existente en torno a la divulgación voluntaria de información sobre $\mathrm{Cl}$ que se canaliza a través del RI, aportando evidencia de la relación entre la concepción clásica del Cl y sus tres dimensiones, y el enfoque del RI del IIRC. En este sentido, el trabajo supera una de las principales críticas a la literatura existente que, en su mayoría, ha considerado únicamente a los EF como fuente de información sobre Cl, obviando el análisis de otros informes. Además, este estudio emplea la metodología de análisis de datos textuales (Franzosi, 2010; Vaara, 2010), que permite el estudio multidimensional de textos no estructurados; diferenciándose del tradicional análisis de contenido, que es de uso habitual en los es- 
tudios sobre la divulgación de información de Cl y que ha sido objeto de diversas críticas.

De esta forma, el trabajo también resulta superador de algunos cuestionamientos metodológicos realizados a las investigaciones previas. Pero, a la vez, la adopción de la metodología de análisis de datos textuales le confiere originalidad, porque no se han encontrado antecedentes de su utilización dentro de esta línea de investigación ni dentro del ámbito de la contabilidad financiera en general, siendo escasos los trabajos que aplican este tipo de análisis en el campo de las Ciencias Económicas? ${ }^{1}$. Finalmente, el trabajo también se puede justificar desde el punto de vista del contexto, pues se realiza sobre empresas cotizantes en el mercado de capitales argentino, donde no se han constatado trabajos publicados de esta naturaleza.

El trabajo se estructura de la forma que se expone a continuación. Después de esta introducción se presenta el desarrollo del trabajo dividido en cuatro secciones. La primera de ellas aborda el marco conceptual del Cl y sus dimensiones. En la segunda se exponen las principales características del enfoque del RI y su relación con la concepción clásica del Cl. En la tercera se describen los aspectos metodológicos del estudio empírico realizado y, en la cuarta sección, se presentan los resultados de dicho estudio y su discusión. Para finalizar, se exponen los comentarios finales, las limitaciones de la investigación y sus perspectivas.

\section{EL CAPITAL INTELECTUAL Y SUS COMPONENTES}

Tal como se indicó en la introducción, el Cl de la empresa refiere al conjunto de elementos intangibles, entre los que destaca el conocimiento, que contribuye a la ventaja competitiva sostenible de la firma y a la creación de valor. Se trata, de este modo, de un concepto que abarca todas las formas de elementos intangibles (Cañibano, Sánchez, García y Chaminade, 2002), que la mayor parte de los autores coincide en agrupar en tres grandes componentes o dimensiones: capital humano (CH), capital estructural (CE) y capital relacional (CR).
EI CH refiere al conocimiento tácito (Bontis, 1998), que reside en los empleados e incluye los saberes, capacidades, experiencias y habilidades que poseen los mismos (Cañibano y cols., 2002). Por su parte, el CE es la infraestructura que incorpora, forma y sostiene al CH (Edvinsson y Malone, 1997) y que permite optimizar su rendimiento (Bontis, 1998). Incluye rutinas organizativas, procedimientos y sistemas que permiten la conversión del conocimiento individual en colectivo (Bollen, Vergauwen y Schnieders, 2005), aunque involucra también los elementos que aluden a la capacidad de renovación de la empresa y los resultados de la innovación (Ochoa Hernández, Prieto Moreno y Santidrián Arroyo, 2010), algunos de cuales "pueden protegerse legalmente y convertirse en derechos de propiedad intelectual o industrial, como los derechos de autor o las patentes" (Cañibano y cols., 2002, p. 19).

La amplia variedad de componentes del CE ha motivado la distinción entre capital organizacional y capital de renovación y desarrollo, tal como lo proponen Edvinsson y Malone (1997) y Roos, Roos, Dragonetti y Edvinsson (2001). El CR refiere a la red de relaciones de la empresa con diferentes stakeholders (Roos y cols., 2001) y representa una dimensión esencialmente externa a la empresa, que incluye también los nombres de sus productos, las marcas y su reputación o imagen (Ficco, 2020a). La Figura 1 esquematiza las distintas dimensiones del $\mathrm{Cl}$.

Es importante mencionar que diferentes autores al abordar la temática de las relaciones de la organización, en el marco conceptual del Cl, han destacado la relevancia del capital social (Ficco, 2020b). Algunos como Nahapiet y Ghoshal (1998), lo consideran como un componente autónomo que incluye la red de relaciones que se da entre los miembros de la organización, las relaciones que posee la organización como unidad social y los recursos que se originan a partir de esas relaciones. Otros autores como Bueno y cols. (2008), lo reconocen como una categoría incluida dentro del CR que abarca el conjunto de relaciones que mantiene la empresa con los Stakeholders, que no están directamente relacionados con el ámbito del negocio, como podrían ser las administraciones públicas o la sociedad en general, y que tienen que ver con el compromiso social, el desarrollo econó-

\footnotetext{
1. En el ámbito de las Ciencias Económicas, el análisis de datos textuales se ha utilizado, por ejemplo, en el estudio de los discursos empresariales (Benites Lazaro, Mello Théry \& Lahsen, 2017) y las percepciones de los consumidores (Goulart, Viana \& Lucchese-Cheung, 2020); y en revisiones bibliográficas (Avelar, da Silva Oliveira \& da Silva Pereira, 2019). No obstante, no se han encontrado antecedentes de su aplicación dentro de la línea de investigación enfocada en el análisis de la divulgación voluntaria de Cl, ni tampoco en las investigaciones que se desarrollan dentro del campo de la contabilidad financiera.
} 


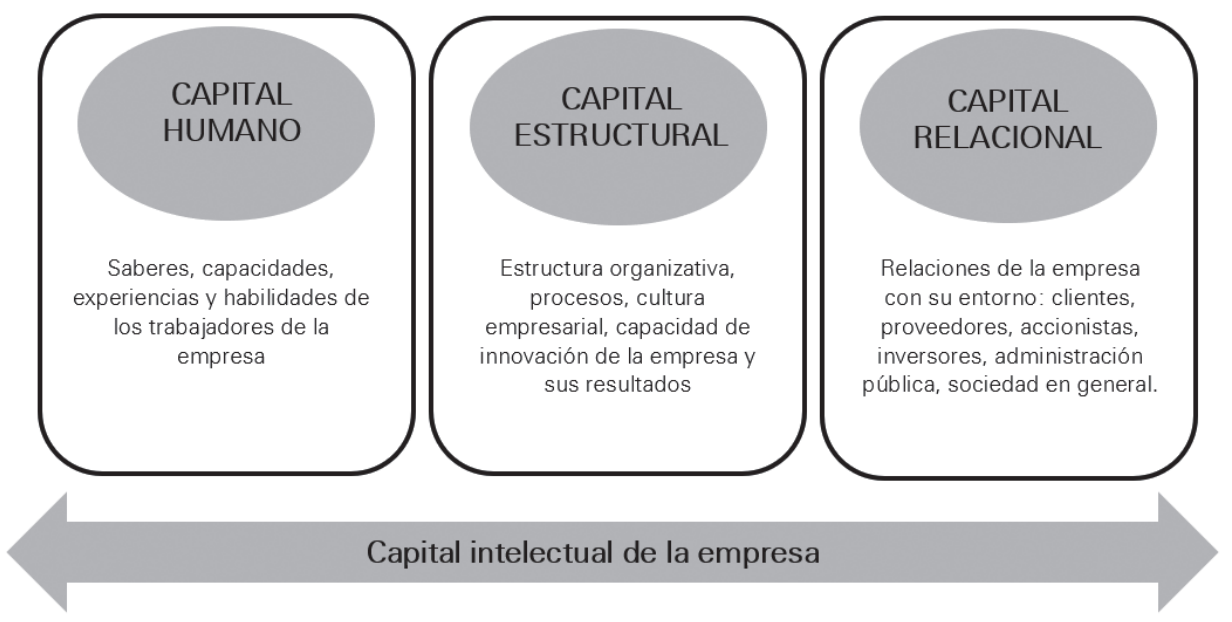

Figura 1. Dimensiones del capital intelectual. Fuente: Ficco (2020b).

mico del entorno, la defensa del medio ambiente, la reputación corporativa, entre otras.

La Tabla 1 muestra las distintas dimensiones del
Cl y los diferentes componentes o elementos que integran cada dimensión, de acuerdo con la propuesta de los autores de esta investigación.

Tabla 1. Dimensiones del Cl y componentes o elementos que integran cada dimensión

\begin{tabular}{|c|c|c|}
\hline Capital Humano & Capital Estructural & Capital Relacional \\
\hline - Nivel educativo & - Estructura organizativa & - Nombres de los productos \\
\hline - Saber hacer (know how) & - Cultura organizacional & - Marcas \\
\hline - Calificación profesional & - Procesos & - Imagen de la empresa \\
\hline - Conocimiento profesional & - Modelo de gestión & - Bases de datos de clientes \\
\hline - Experiencia previa & - Gobierno corporativo & - Lealtad y la satisfacción de los clientes \\
\hline - Capacidad para aprender & - Sistemas de información y de & - Bases de datos de proveedores \\
\hline - Capacidad para innovar & 年 & - Pactos con los proveedores \\
\hline - Capacidad emprendedora & & - Acuerdos de cooperación \\
\hline - Creatividad & ctomeco rodecinformútiç & - Alianzas estratégicas \\
\hline - Capacidad de negociación & 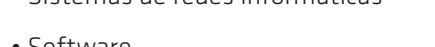 & - Poder comercial \\
\hline - Capacidad para trabajar en equipo & 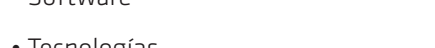 & - Capacidad de negociación con instituciones \\
\hline $\begin{array}{l}\text { - Flexibilidad del empleado (habili- } \\
\text { dad para cambiar) }\end{array}$ & - Flexibilidad organizativa & - Compromiso social \\
\hline - Habilidad para actuar & - Capacidad organizativa de aprender & - Defensa del medio ambiente \\
\hline - Motivación & - Esfuerzos de I+D & - Reputación corporativa \\
\hline - Satisfacción & - Propiedad Intelectual & - Posicionamiento en el mercado \\
\hline - Lealtad & - Patentes & \\
\hline \multicolumn{3}{|l|}{ - Rendimiento } \\
\hline \multicolumn{3}{|l|}{ - Diversidad cultural } \\
\hline - Salud y seguridad & & \\
\hline
\end{tabular}

Fuente: Bueno y cols. (2008), Cañibano y cols. (2002), Lentjušenkova y Lapina (2016) y elaboración propia. 
Diversas iniciativas se han desarrollado en pos de generar propuestas de informes de $\mathrm{Cl}$, entendidos como "informes o estados que expliquen el qué, para qué y cómo se ha llevado a cabo la identificación y medición de los activos intangibles que componen el citado concepto de Capital Intelectual" (Bueno y cols., 2011, p. 70). Sin embargo, ninguna de estas propuestas se aplica de manera generalizada ni tampoco han logrado un consenso, a nivel global, que haya implicado su estandarización. Sí han surgido iniciativas tendientes a poner énfasis en la consideración de información no financiera (como los estándares del Clobal Reporting Initiative (GRI), las normas del Sustainability Accounting Standards Board (SASB) y el marco del informe integrado del IIRC, el cual se encuentra estrechamente vinculado a elementos intangibles clave de la generación de valor público y organizacional, en tanto abarca un amplio abanico de temas sociales, medioambientales y otros relacionados con la sostenibilidad (Ficco y Sader, 2020).

Por ello, en el ámbito empresarial la alternativa más utilizada para dar a conocer el Cl, en caso de estimarlo adecuado, es la de divulgar esta información de forma totalmente voluntaria, usando para ello diversos formatos y canales, entre los que destacan la información complementaria a los EF, las memorias de sostenibilidad y los informes integrados.

\section{EL REPORTE INTEGRADO}

\section{El enfoque del Reporte Integrado y su estructura}

Actualmente, esta iniciativa es liderada por el IIRC, que presentó en 2013 el Marco Internacional del Reporte Integrado (MIRI), para su elaboración. EI MIRI establece que "un informe integrado es una comunicación concisa sobre cómo la estrategia, gobernanza, rendimiento y perspectivas de la empresa, en el contexto de su entorno conduce a la creación de valor sobre el corto, mediano y largo plazo" (IIRC, 2013, p. 8). En el mismo sentido, en su párrafo 1.7, enuncia que el objetivo principal del RI "es explicar a los proveedores de capital financiero cómo la organización crea valor a lo largo del tiempo" (IIRC, 2013, p. 8), incluyendo, por lo tanto, información de carácter financiero y no financiero.

En este sentido, Giraldo López, Soto Sossa y Mejía Delgado (2018), destacan que el objetivo principal del RI se enfoca en la generación de valor y que, para comprender esta situación, es importante aclarar que "la compañía no solo crea valor aumentando su liquidez y siendo más productiva, sino que también crea valor cuando tiene impactos positivos en los factores externos como el económico, social y ambiental" (p. 170).

En lo que respecta a los usos del informe integrado, el MIRI señala que este reporte "beneficia a todos los grupos interesados en la habilidad de la organización de crear valor a lo largo del tiempo, incluyendo empleados, clientes, proveedores, socios, comunidad local, legisladores, reguladores y políticos" (IIRC, 2013, p. 8). Sin embargo, desde el punto de vista ontológico, existe disidencia entre diferentes autores. Por un lado, algunos consideran que más allá de la apertura de información, los RI están dirigidos a los inversores, por lo que se centran en la creación de valor para los accionistas y no para la sociedad, relegando cuestiones medioambientales y de sostenibilidad (De Villiers, Rinaldi y Unerman, 2014). Y por otro, existen quienes destacan su utilidad para los stakeholders, señalando que brindan una imagen holística con vistas al futuro, abarcando no solo el campo financiero, sino también cuestiones sociales y ambientales (Navarrete y Gallizo, 2018).

Con respecto al proceso de creación de valor, el MIRI considera que el valor creado por una empresa no se genera en forma solitaria, sino más bien por la interrelación de factores externos: la situación económica, tecnologías, aspectos sociales, clientes, proveedores, capital humano, etc. Así, define a la creación de valor como "el proceso que se traduce en aumentos, disminuciones o transformaciones de los capitales causados por las actividades y la producción de la organización" (IIRC, 2013, p. 36). Estos capitales se refieren a los "inventarios de valor de los que depende el éxito de cualquier empresa ya que, de una forma u otra, son recursos de su modelo de negocio, y (...) se transforman a través de las actividades (...) de la organización" (IIRC, 2013, p. 35). Estos capitales se clasifican, tal como establece el MIRI (IIRC, 2013), de la siguiente forma:

Capital Financiero: fondos a los que el ente accede para la elaboración de sus productos o prestaciones de servicios, incluidos los fondos obtenidos de fuentes externas (deuda).

- Capital Financiero: fondos a los que el ente accede para la elaboración de sus productos o prestaciones de servicios, incluidos los fondos obtenidos de fuentes externas (deuda).

- Capital Intelectual: intangibles basados en el 
conocimiento, como propiedad intelectual y capital organizacional.

- Capital Humano: competencias, capacidades y experiencia de las personas.

- Capital Social y Relacional: instituciones y relaciones; como así también, la capacidad de compartir información para mejorar el bienestar individual y colectivo.

- Capital Natural: todos los recursos renovables y no renovables que el ente utiliza para la elaboración de sus productos o prestaciones de servicios, y los procesos medioambientales que sostienen la prosperidad de la organización.

Estos capitales se encuentran en continua interacción y transformación. Tal como se indica en el párrafo 2.12 del MIRI: "existe un flujo constante entre y dentro de los capitales, a medida que aumentan, disminuyen o se transforman" (IIRC, 2013, p. 12). Con relación a esta cuestión, y con la forma en que debe ser presentada la información, Sánchez, Rivera y Brand (2019), destacan que "el enfoque estratégico y la orientación futura hacen referencia a que los contenidos del RI y su presentación deben estructurarse de forma articulada para cada tipo de capital" (p. 30).

Con respecto a la presentación del RI, aunque el MIRI aclara que no establece indicadores clave de desempeño (Key Performance Indicators), propone una serie de principios guías (Directrices) y contenidos clave (Contenidos) para la preparación del informe, de modo tal que el RI resulte susceptible de ser conceptuado como elaborado de conformidad con dicho marco.

En tanto, los principios que deben ser utilizados como guía para la presentación y formulación del reporte se usarán tanto de manera individual como colectiva, porque la información que se presenta debe ser interrelacionada. Estos principios se reflejan en directrices que refieren tanto a aquellos aspectos que se espera sean exteriorizados, como a las características que debe reunir el informe, a saber: Enfoque estratégico y orientación futura (comunicación de la estrategia y su vínculo con la creación de valor); Conectividad de la información (visión holística); Relación con los grupos de interés (calidad de las relaciones); Materialidad (asuntos que afecten la habilidad de crear valor); Concisión (conciso y suficiente); Fiabilidad y exhaustividad; Confiabilidad e integridad (sobre bases que permitan su comparación).
Referente a los contenidos, el MIRI (IIRC, 2013) propone ocho que responden a las siguientes preguntas:

Descripción general de la organización y de su entorno externo: ¿Qué es lo que hace la organización y cuáles son las circunstancias en las que opera?

- Gobierno Corporativo: ¿Cómo funciona la estructura de gobierno corporativo en la organización para apoyar su habilidad de crear valor a corto, medio y largo plazo?

- Modelo de negocios: ¿Cuál es el modelo de negocios de la organización?

- Riesgos y oportunidades: ¿Cuáles son los riesgos y oportunidades que afectan la capacidad de la organización para crear valor en el corto, medio y largo plazo, y cómo les hace frente la organización?

- Estrategia y asignación de recursos: ¿Hacia dónde quiere ir la organización y cómo piensa llegar?

- Desempeño: ¿Hasta qué punto tiene la organización que haber alcanzado sus objetivos estratégicos en un determinado período y cuáles son sus resultados en términos de efectos en los capitales?

- Perspectivas: ¿Qué desafíos e incertidumbres puede encontrar la organización implementando su estrategia y cuáles son las implicancias potenciales para su modelo de negocio y desempeño futuro?

- Base para la preparación y presentación del informe: ¿Cómo determina la organización qué asuntos deberían ser incluidos en el RI y cómo esos asuntos son cuantificados o evaluados?

La Figura 2 esquematiza los Principios enunciados por el MIRI y los Contenidos del RI, a modo de respuesta a las siguientes preguntas: ¿cómo se presenta la información en un RI? y ¿qué información debe contener?

De esta forma, y como sintesis de lo expresado hasta aquí, es posible afirmar que el RI brinda información acerca de los distintos capitales considerados como los recursos y las relaciones utilizados y afectados por una organización, con la finalidad de explicar cómo la organización interactúa con el entorno externo y con esos capitales para crear valor. 


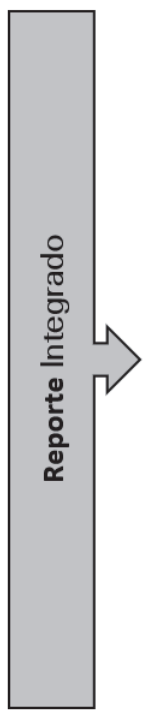

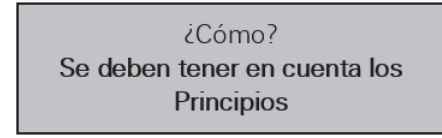

- Enfoque estratégico

- Conectividad de la información

- Relación con los stakeholders

- Materialidad

- Concisión

- Fiabilidad y exhaustividad

- Confiabilidad e integridad
¿Qué?

Contenidos

- Visión del conjunto organizacional y del entorno

- Gobierno

- Modelo de negocios

- Riesgos y oportunidades

- Estrategia y asignación de recursos

- Desempeño

- Perspectivas (desafíos)

- Bases para la preparación y presentación

Figura 2. Principios y Contenidos del RI. Fuente: elaboración propia.

Finalmente, resulta importante hacer referencia a la utilización del RI en la práctica empresarial, como "nueva forma emergente de reportes corporativos" (Wild y van Staden, 2013, p. 3). En este sentido, Frías-Aceituno, Rodríguez-Ariza y García-Sánchez (2013), revelan que las empresas de mayor tamaño y mayor cantidad de miembros en su gobierno corporativo son las que tienden a implementar mejores prácticas, más objetivas y comparables. Asimismo, algunos estudios han mostrado que las guías del GRI han contribuido a mejorar la práctica de los RI, porque las empresas que están más familiarizadas con las mismas son las que demuestran haber entendido mejor su lógica (Macias y Farfan-Lievano, 2017; Dumay y cols., 2016). No obstante, falta todavía un largo camino por recorrer para su armonización y utilización generalizada, pero la investigación está avanzando (Dumay, Bernardi, Guthrie y Demartini, 2016) y se enfrenta al desafío de desarrollar políticas y prácticas que conduzcan a la presentación de RI más sólidos y eficaces (De Villiers y cols., 2014).

\section{El capital intelectual en el Reporte Integrado}

Aunque la presentación de informes específicos sobre $\mathrm{Cl}$ se ha vuelto prácticamente inexistente ${ }^{2}$, el Cl se ha reencarnado en el movimiento emer- gente del RI (Abhayawansa, Guthrie y Keynes, 2019), y se podría invocar al Darwinismo para argumentar que dicha extinción sería una evidencia de que la contabilidad del Cl no es adecuada para su propósito. Sin embargo, Abhayawansa y cols. (2019), muestran como contraargumento que la resurrección del $\mathrm{Cl}$ en el marco del RI, da testimonio de la capacidad de la contabilidad para adaptarse a los cambios, circunstancias y lógicas institucionales. Y esta adaptación produce modificaciones en las prácticas y en la presentación de informes, las que, siguiendo a Rivera Arrubla y cols. (2016), surgen porque los distintos interesados demandan informes que permitan una rendición de cuentas más transparente e integral y que va más allá de las necesidades de los proveedores de capital financiero, quienes eran considerados prioritarios en los informes tradicionales.

Con respecto a lo anterior, el estudio de Terblanche y De Villiers (2019), revela que los RI conducen a una mayor divulgación de los elementos del Cl y a un mayor énfasis sobre el mismo en el informe. Según Ramin (2013), estos informes son útiles para describir el pasado y desempeño futuro de una organización, informando sobre las 3P del management (People, Process and Products), las que se relacionan estrechamente con los tres pilares o componentes del Cl: CH, CE y CR.

\footnotetext{
2. En la actualidad es difícil encontrar alguna empresa que cotice en el mercado de valores, en cualquier parte del mundo, que todavía elabore el informe de CI para su ámbito externo (Dumay, 2016), situación que Abhayawansa y cols. (2019) atribuyen al surgimiento del RI.
} 
De este modo, acreditando la idea de que el RI emerge como una manifestación de la capacidad de adaptación de la contabilidad que ha reivindicado la importancia del $\mathrm{Cl}$, en sus dimensiones humana, estructural y relacional, interesa analizar la relación entre la concepción clásica de creación de valor del enfoque de $\mathrm{Cl}$ con la del marco del RI.
Y, más concretamente, identificar los componentes de los capitales que se describen en el MIRI y efectuar una asociación entre ellos y las tres dimensiones en las que habitualmente se engloban los componentes del $\mathrm{Cl}$, a saber: CH, CE y CR. Para reflejar estas relaciones se construye la Tabla 2.

Tabla 2. Los capitales del RI y las dimensiones del Cl en su concepción clásica.

\begin{tabular}{|c|c|c|c|}
\hline \multirow{2}{*}{$\begin{array}{l}\text { Capitales } \\
\text { del MIRI }\end{array}$} & \multicolumn{3}{|c|}{ Dimensiones del $\mathrm{Cl}$} \\
\hline & $\begin{array}{c}\text { Capital } \\
\text { Humano }(\mathrm{CH})\end{array}$ & Capital Estructural (CE) & Capital Relacional (CR) \\
\hline Capital Humano & $\begin{array}{l}\text { - Competencias, capacida- } \\
\text { des y experiencia de las } \\
\text { personas } \\
\text { - Lealtad y motivación } \\
\text { - Alineación y apoyo al } \\
\text { modelo de gobierno, } \\
\text { gestión y valores éticos } \\
\text { de la organización } \\
\text { - Capacidad para compren- } \\
\text { der, desarrollar e im- } \\
\text { plementar la estrategia } \\
\text { organizacional } \\
\text { - Capacidad para dirigir, } \\
\text { gestionar y colaborar }\end{array}$ & & \\
\hline $\begin{array}{c}\text { Capital } \\
\text { Intelectual }\end{array}$ & & $\begin{array}{l}\text { - Propiedad intelectual: } \\
\text { patentes, derechos de } \\
\text { autor, software, derechos } \\
\text { y licencias } \\
\text { - Capital organizacional: } \\
\text { conocimiento tácito, } \\
\text { sistemas, procedimientos } \\
\text { y protocolos }\end{array}$ & \\
\hline $\begin{array}{l}\text { Capital Social y } \\
\text { Relacional }\end{array}$ & & & $\begin{array}{l}\text { - Relaciones con grupos de interés clave } \\
\text { - Capacidad de compartir información } \\
\text { - Normas compartidas } \\
\text { - Valores y conductas comunes } \\
\text { - Marca } \\
\text { - Reputación }\end{array}$ \\
\hline Capital Natural & & & - Salud del ecosistema \\
\hline
\end{tabular}

Fuente: IIRC (2013) y elaboración propia.

En la Tabla 2 se advierte una fuerte asociación entre las dimensiones conceptuales del $\mathrm{Cl}$ y los componentes de los Capitales Humano, Intelectual y Social y Relacional que se describen en el MIRI. Así, el CI del MIRI, que reúne los inventarios de valor asociados a los intangibles basados en el conocimiento de la organización, se relaciona a la dimensión estructural del Cl. Del mismo modo, es posible identificar que los Capitales Humano y Social y Relacional se encuentran asociados conceptualmente a las dimensiones del $\mathrm{Cl}$ que reciben análogas denominaciones.
Por otro lado, en la Tabla 2 se hace evidente la ausencia de algunas de las categorías de capital descritas por el MIRI. Ello se debe a que el modelo de creación de valor que presenta incluye, además, elementos de carácter tangible considerados necesarios para la producción de bienes o la prestación de servicios, como es el caso de los bienes inmovilizados y la infraestructura (Capital Industrial), junto a los recursos financieros (Capital Financiero) y a los recursos naturales renovables y no renovables (Capital Natural), que no estarían involucrados en la concepción clásica del 
$\mathrm{Cl}$, por englobar elementos de carácter intangible.

Finalmente, y en relación con el Capital Natural, este incluye, además de los recursos naturales, procesos medioambientales que sí pueden asociarse a la dimensión estructural del $\mathrm{Cl}$, en tanto resultan representativos del compromiso de la organización con el medio ambiente.

Se aprecia, de esta manera, la complementariedad existente a nivel teórico de ambos enfoques: la concepción clásica que incluye tres dimensiones para el Cl, y el soporte conceptual del RI, lo que deja en claro la idea que esbozábamos al comienzo de esta sección respecto de que el marco del RI ha reivindicado la importancia del $\mathrm{Cl}$ en los procesos de creación de valor de las organizaciones. En ese sentido, el RI se presenta como una importante estrategia de revelación de información sobre $\mathrm{Cl}$, que puede ser usada por la gerencia de la empresa para ayudar a reducir las asimetrías informativas y generar confianza en el mercado (Dumay, La Torre y Farneti, 2019), teniendo en cuenta que algunos estudios previos han demostrado que la información sobre $\mathrm{Cl}$, voluntaria y no financiera, influye positivamente en los inversores (Beretta y cols., 2019).

\section{ASPECTOS METODOLÓGICOS DEL ESTUDIO EMPÍRICO}

Siguiendo la clasificación de Hernández, Fernández y Baptista (2014), el estudio empírico es de tipo exploratorio, en tanto propone analizar la divulgación de información sobre $\mathrm{Cl}$ en el RI desde una perspectiva novedosa, que busca mostrar la complementariedad de ambos enfoques a través de la aplicación de una metodología que también resulta original: el análisis de datos textuales.

El análisis se realiza sobre empresas listadas en el mercado de capitales argentino que han publicado su RI elaborado, a partir del marco de referencia del IIRC. Solo tres empresas pertenecientes al sector bancario cumplen actualmente con esta condición y, en particular, dos de ellas lo hacen desde el año 2018 (la tercera empresa pública su RI -elaborado bajo el marco del IIRC- desde 2014) ${ }^{3}$. Por ello, el período de análisis elegido es 2018-2019, el cual tiene como año de inicio el 2018, para poder contar con información de las tres empresas para todo el período, y finaliza en 2019, por ser este el último ejercicio cerrado al momento de realizar la investigación.

De este modo, en lo que respecta a la dimensión temporal, la investigación es de tipo longitudinal, porque los datos se recolectan en distintos momentos dentro del período de tiempo seleccionado para el análisis (Hernández y cols., 2014).

El reducido número de empresas listadas que elaboran RI y el período de tiempo seleccionado, reflejan el desarrollo incipiente de los RI en Argentina, situación que, como se indicó en la introducción, también se evidencia en otros países latinoamericanos (Rivera Arrubla y cols., 2016; Macias y Farfan Lievano, 2017). Por otra parte, la predominancia del sector bancario en las empresas que elaboran RI en Argentina es consistente con el estudio de Wild y van Staden (2013), sobre las prácticas emergentes de RI publicados en la base de datos del IIRC, donde se detecta que los RI pioneros no pertenecían mayoritariamente a sectores con alto impacto social y ambiental, sino que al sector financiero.

El estudio empírico fue realizado sobre la base de la metodología de análisis de datos textuales, entendiéndola como el conjunto de métodos de análisis multidimensionales exploratorios sobre datos lingüísticos (Franzosi, 2010). Esta metodología integra herramientas estadísticas con técnicas cualitativas de análisis basadas en la interpretación, para explorar las conexiones entre las palabras o formas utilizadas en un conjunto de textos o corpus, referidos a un objeto de estudio específico (Vaara, 2010).

En este sentido, los RI fueron considerados como datos textuales, cuyo contenido permitió indagar sobre la forma en que la información referida al Cl es comunicada en el marco de referencia del IIRC. El corpus o conjunto de textos analizados consistieron en las transcripciones de seis RI correspondientes al período sujeto a estudio, publicados por las empresas que conforman la población analizada. Los RI se obtuvieron de las páginas web correspondientes a cada empresa sujeta a estudio.

El análisis de datos textuales se realizó en dos etapas. En la primera, se pretendió identificar las estructuras temáticas subyacentes de los RI, con la finalidad de efectuar una asociación entre las mismas y las dimensiones del CI (CH, CE y CR), a partir de su contenido. Para ello, se utilizó el software IRAMuTeQ (Interfaz de R para el Análisis Multidimensional de los Textos y Cuestionarios),

3. Grupo Financiero Galicia S.A. y Banco Patagonia S.A. adoptaron el marco del IIRC para informar su desempeño económico, social y ambiental a partir de 2018. Por su parte, Banco Macro S.A. hizo lo propio en 2014, siendo la primera empresa en presentar un RI en Argentina. 
el cual permite realizar análisis multidimensionales de textos no estructurados. Concretamente, se aplicaron los métodos de Clasificación Jerárquica Descendente (CJD) y el análisis de similitud.

El método de CJD, propuesto por Reinert (1983), permite obtener una representación sintética del conjunto de textos analizados a partir de su subdivisión en clases lexicales o clusters, construidos según la frecuencia de ocurrencia de las palabras y su ubicación en el corpus. Cada clase se conforma por segmentos de textos que presentan un vocabulario similar entre sí, y diferente de los segmentos de otras clases (Camargo y Justo, 2013). Este método parte de la premisa de que las palabras no son independientes unas de otras, sino que reflejan temas subyacentes (Reinert, 1983).

En ese sentido, las clases lexicales representan temáticas divulgadas en los RI que pueden ser descritas por el vocabulario que las define. Complementariamente, el análisis de similitud identifica las conexiones que existen dentro del texto analizado, a partir de las co-ocurrencias entre las palabras (Camargo y Justo, 2013). De este modo, este método fue aplicado a los subcorpus conformados por los segmentos de textos que integran cada clase lexical identificada a partir de la CJD, con la finalidad de obtener una representación gráfica de la estructura de su contenido y las relaciones entre las palabras asociadas a cada clase.

Luego, en una segunda etapa se buscó profundizar la indagación con relación al contenido de la información sobre Cl divulgada en los RI, a través del análisis de contenido temático. El análisis de contenido (Bardin, 1996), constituye un método ampliamente utilizado para investigar la divulgación de información sobre $\mathrm{Cl}$ en diversos medios, aunque, como se indicó en la introducción, la mayor parte de los estudios previos ha considerado únicamente a los EF, obviando el análisis de otros informes (Dumay y Cai, 2014; Castilla y Ruiz, 2017). Siguiendo a Abhayawansa, Aleksanyan y Cuganesan (2018), el primer paso para el análisis temático consistió en la lectura de los RI, y, de esta forma, lograr comprender los textos en su conjunto. A partir de la lectura activa de los RI se identificaron palabras, frases o fragmentos dentro de los datos analizados, relacionados al marco conceptual del $\mathrm{Cl}$, los cuales fueron segmentados en categorías y subcategorías de acuerdo con la temática subyacente en los mismos.

\section{RESULTADOS Y DISCUSIÓN}

\section{Clasificación Jerárquica Descendente (CJD) y aná- lisis de similitud}

El corpus conformado por el conjunto de RI analizado fue dividido a través del método de CJD, en cuatro clases lexicales o clusters. En la Figura 3 se presenta el dendrograma de clases que ilustra las particiones hechas en el corpus hasta llegar a las clases finales, y se indica el porcentaje de segmentos de textos que conforman cada clase junto a las palabras significativamente asociadas a cada una.

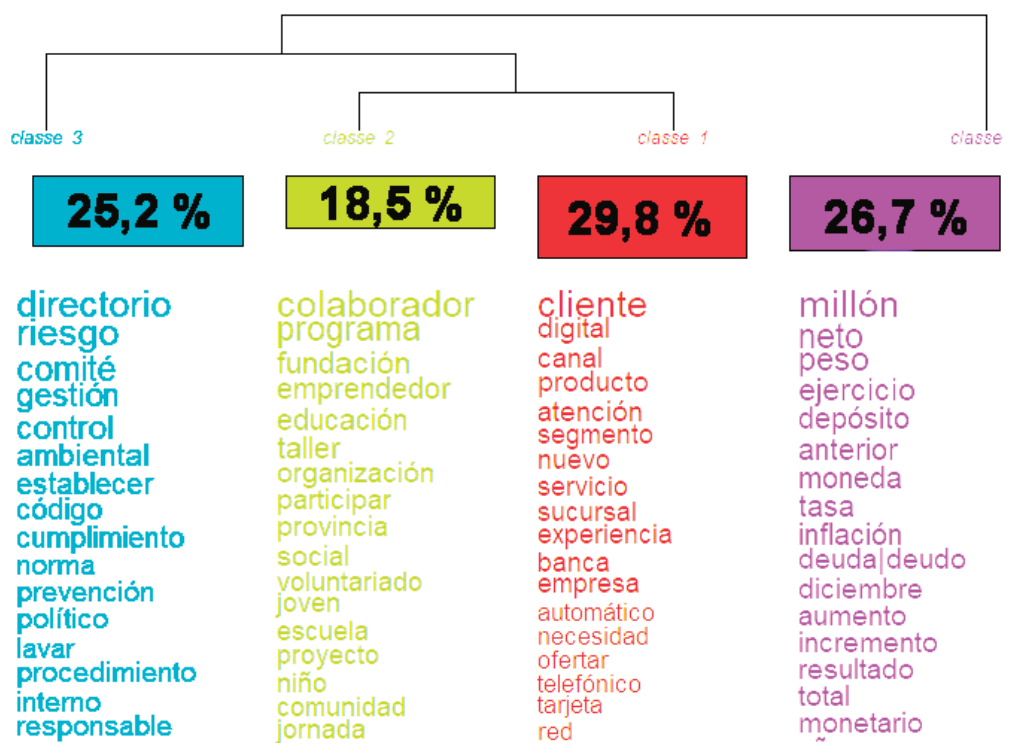

Figura 3. Estructura temática de los RI. Fuente: elaboración propia. 
La CDJ permitió identificar las estructuras temáticas subyacentes en los RI a través de la construcción de clases lexicales. Esto se complementó con el análisis de similitud al interior de cada una de las clases, para evidenciar co-ocurrencias entre las palabras asociadas a las mismas. El análisis en conjunto de estos dos métodos, sumado a la posibilidad de recuperar el contexto de uso de las palabras estadísticamente significativas y con mayor frecuencia de ocurrencia que conforman cada clase a través del software IRAMuTeQ, permitieron inferir las temáticas subyacentes en cada clase lexical, las que luego fueron contrastadas con las categorías de capitales del MIRI y las dimensiones del $\mathrm{Cl}$.

La clase 1 es la principal clase lexical del corpus,

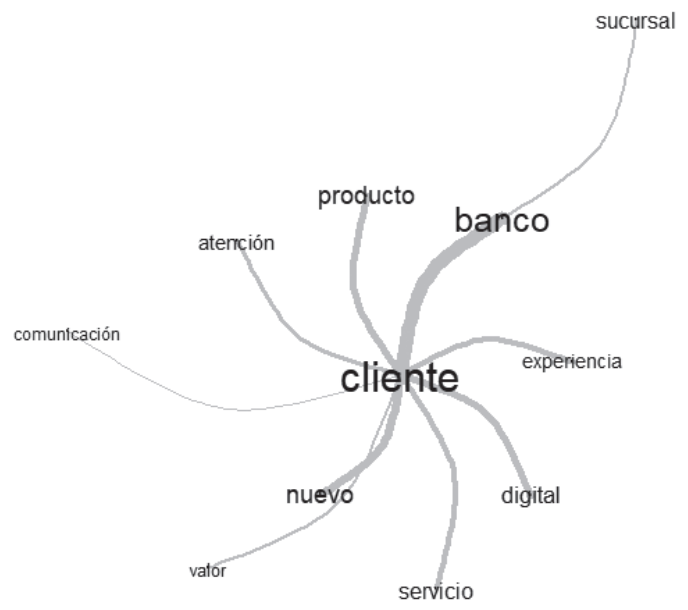

Figura 4. Análisis de similitud de las clases lexicales 1 y 2.

La clase 2 , siendo la clase minoritaria (18.52\%), hace referencia a aspectos relacionados con el componente humano de los bancos (colaborador). El análisis de similitud (Figura 4) asocia a esta palabra aspectos referidos a capacitación y formación de los colaboradores, capacidad de trabajo en equipo y liderazgo (Capital Humano). También es posible identificar, en esta clase, palabras asociadas a la relación de los bancos con la comunidad a través de fundaciones y el desarrollo de distintos programas sociales (Capital Social y Relacional).

La clase 3 (25.24\% de los segmentos analizados) se refiere a la responsabilidad social de los ban- conformada por el $29.56 \%$ de los segmentos de texto analizados. Esta clase agrupa palabras relacionadas a los productos y servicios ofrecidos por las entidades financieras a distintos segmentos de clientes y a sus canales de distribución. En el análisis de similitud (Figura 4), se destaca la centralidad de la palabra cliente, dada su significatividad estadística y frecuencia de uso. En la Figura 4 se observa que el cliente se asocia a la provisión de productos y servicios financieros a través de la red de sucursales de los bancos y otros canales de atención (Capital Industrial). Por otro lado, también se relaciona con cliente, el diseño y medición de la experiencia de los mismos y el desarrollo de soluciones digitales que les proporcionen valor (Capital Intelectual).

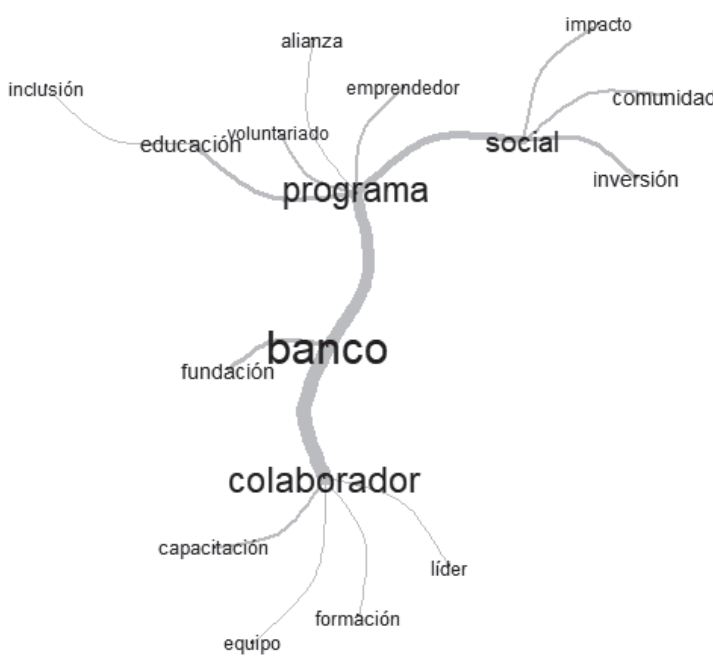

cos frente a sus stakeholders. Como se observa en la Figura 5, la palabra central de esta clase es banco, el cual, por medio de su órgano de administración (directorio, gerencia), debe dar cuenta del desempeño económico, financiero, social y ambiental a sus accionistas y otros stakeholders, a través de los RI (Capital Social y Relacional). En efecto, los bancos deben desarrollar sistemas de información y de control (Capital Intelectual) que permitan una adecuada gestión de riesgos, particularmente aquellos relacionados al cumplimiento de sus objetivos y al impacto ambiental (Capital Natural). 

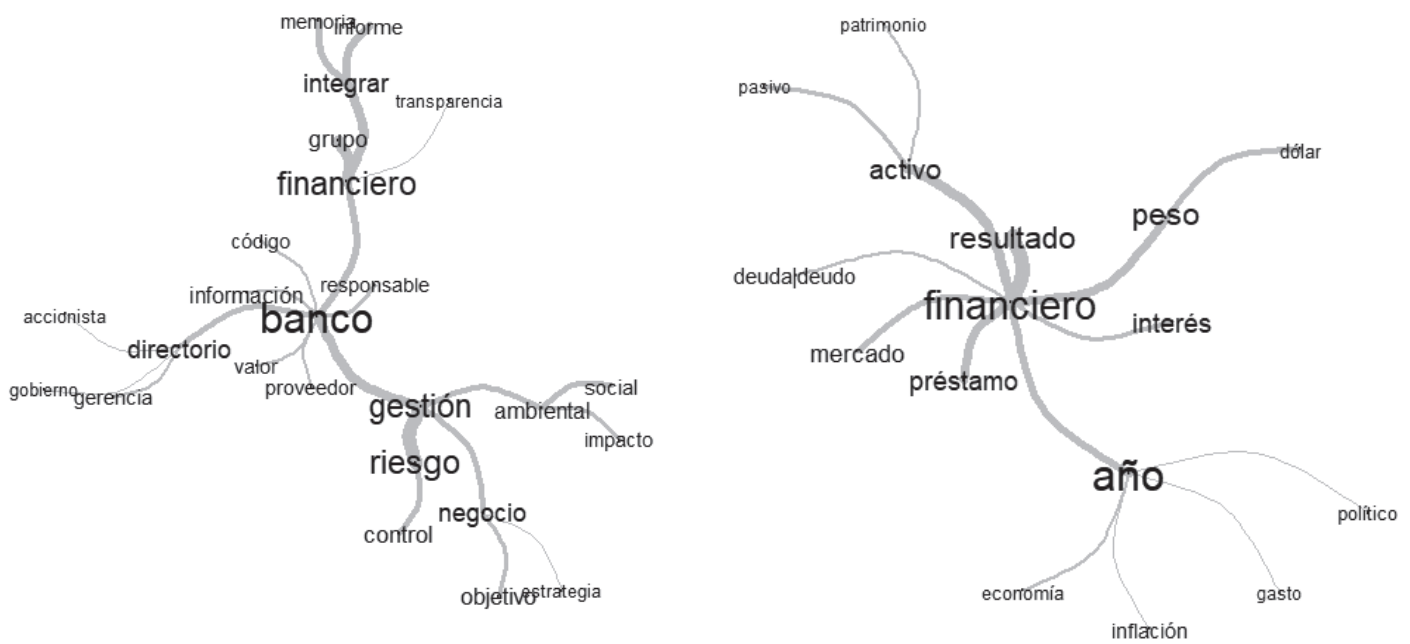

Figura 5. Análisis de similitud de las clases lexicales 3 y 4 . Fuente: elaboración propia.

Por último, la clase 4, segunda en orden de importancia, está conformada por el $26.68 \%$ de los segmentos de texto. Esta clase, tal como surge de sus palabras asociadas y del análisis de similitud (Figura 5), hace clara referencia a variables del contexto (economía, inflación, político, mercado, tasa de interés, dólar, entre otras) que influyeron en el desempeño económico y financiero de los bancos (Capital Financiero).

De este modo, los métodos de CJD y análisis de similitud permitieron evidenciar que las clases temáticas, en las que se dividieron los RI analizados, responden al esquema de capitales del MIRI. En términos generales, se advirtió que la clase 1 engloba aspectos asociados al Capital Industrial y al Capital Intelectual. La clase 2 abarca temas, principalmente, referidos al Capital Humano. Asimismo, el Capital Social y Relacional está presente en las clases 3 y 2. La clase 4 hace referencia al Capital Financiero. Por su parte, el Capital Natural se hizo presente de manera indirecta en la clase 3. Se evidencia, así, la presencia de los seis capitales del MIRI, destacándose la predominancia de los capitales Industrial, Financiero, Social y Relacional y Humano. La mayor relevancia de estos capitales podría responder al sector de actividad analizado; situación considerada por el IIRC (2013) cuando establece que "no todos los capitales son igualmente relevantes o aplicables a todas las organizaciones" (p.13). Estos resultados son consistentes con los hallazgos de Wild y van Staden (2013), quienes encontraron que el $80 \%$ de las empresas incluidas en la base de datos del IIRC, en febrero de 2013, repor- tó cuatro o más capitales en sus RI.

Además, fue posible advertir la presencia de aspectos relacionados conceptualmente al enfoque del $\mathrm{Cl}$ y sus dimensiones en las clases 1 a 3 , constatando, de este modo, la relación evidenciada desde el punto de vista teórico. Así, el CH se encuentra presente en la clase 2 , en tanto se asocia al conocimiento intrínseco de las personas que conforman el componente humano de los bancos, denominados en los RI como colaboradores, y al desarrollo de sus capacidades y habilidades a través de programas de capacitación y formación. El CE se asocia a elementos involucrados en la clase 1 y la clase 3 , referidos a la capacidad de los bancos de innovar y ofrecer a sus clientes nuevas propuestas de valor, y al desarrollo de sistemas de información y control interno, respectivamente. EI CR, referido a las relaciones entre los bancos y sus distintos stakeholders, puede vincularse con las tres clases mencionadas, a saber: clase 1 (clientes), clase 2 (comunidad) y clase 3 (accionistas, proveedores, comunidad).

Estos resultados están en línea con las evidencias que aportan Melloni (2015), Beretta y cols. (2019) y Salvi y cols. (2020), quienes verificaron, en sus respectivos estudios, la presencia de información sobre CI referida a sus tres dimensiones en los RI. Particularmente, Melloni (2015) advirtió que la mayoría de las divulgaciones sobre $\mathrm{Cl}$ en los RI se enfocaba en la dimensión de CR, lo cual podría explicar la vinculación del CR con tres de las cuatro clases lexicales obtenidas. 


\section{Análisis temático}

En consonancia con el método de análisis propuesto por Vaara (2010), los aportes complementarios de los métodos de CDJ (Figura 3) y análisis de similitud (Figuras 4 y 5) contribuyeron, desde sus fundamentos estadísticos, a determinar el sentido y el modo de uso de las palabras identificadas, aportando sustento empírico a las vinculaciones advertidas desde el punto de vista teórico, entre la concepción clásica del Cl y el soporte conceptual del RI.

No obstante, tal como se indicaba al describir los aspectos metodológicos de este estudio, se aplicó también el análisis temático, en pos de profundi- zar la indagación con relación al contenido de la información sobre Cl divulgada en los RI. Específicamente, con este análisis se busca identificar temas clave que contribuyen a un mejor conocimiento de la forma en que el Cl es revelado a través de dichos informes.

En este marco, a partir de la lectura activa de los RI analizados, se identificó una amplia variedad de temas referidos al Cl, los cuales fueron asociados a las dimensiones conceptuales del mismo (humana, estructural y relacional) y agrupados en categorías, las cuales fueron construidas teniendo en cuenta las temáticas emergentes de los textos. Los resultados de este análisis se exponen en la Tabla 3.

Tabla 3. Temáticas sobre CI divulgadas en los RI analizados.

\begin{tabular}{lll}
\hline \multicolumn{1}{c}{ CAPITAL HUMANO } & \multicolumn{1}{c}{ CAPITAL ESTRUCTURAL } & \multicolumn{1}{c}{ CAPITAL RELACIONAL } \\
\hline - Directorio & - Filosofía empresarial & • Posicionamiento en el mercado \\
- Empleados/Colaboradores & - Estructura organizacional & - Crupo económico y alianzas estratégicas \\
- Selección de personal & - Prácticas de buen gobierno corpo- & - Relaciones con inversores \\
- Formación y desarrollo de compe- & rativo & - Cumplimiento regulatorio \\
tencias & - Sistemas de control y gestión de & - Política de RSC \\
- Política de remuneraciones & riesgos & - Experiencia del cliente \\
- Beneficios al personal & - Cultura organizacional & - Marketing y fidelización \\
- Reconocimientos al personal & - Transformación digital & - Canales de comunicación \\
- Evaluación de desempeño & - Desarrollo de productos y servicios & - Reputación e imagen corporativa \\
- Planes de carrera & - Investigaciones de mercado & - Relaciones con proveedores \\
- Clima laboral & - Sistemas de información & - Política ambiental \\
- Diversidad e inclusión & - Procesos y procedimientos & - Programas de educación e inclusión \\
- Salud y seguridad laboral & - Seguridad de la información y privaci- & social \\
- Comunicación interna & dad de datos & - Fomento al empleo \\
& - Canales de distribución & - Fomento a la cultura y el deporte \\
& & - Acciones en la comunidad
\end{tabular}

Fuente: elaboración propia.

Las categorías temáticas emergentes de los RI permiten constatar empíricamente la presencia de los diferentes componentes o elementos que integran conceptualmente cada dimensión del $\mathrm{Cl}$ y que fueron presentados en la Tabla 1. No obstante, se advierten algunos aspectos que merecen ser destacados en la medida que reflejan la adaptación de la contabilidad del $\mathrm{Cl}$, postulada por Abhayawansa y cols. (2019), al enfoque del $\mathrm{RI}$, centrado en explicar de manera integral "cómo la organización crea valor a lo largo del tiempo" (IIRC, 2013, p. 8).

Respecto del CH, los RI incluyen información referida tanto al personal jerárquico (directores, gerentes), como a sus empleados o colaboradores. Asimismo, los bancos enfatizan la importancia del desarrollo integral de sus colaboradores, 118 ampliando la visión de formación para el trabajo presente implícitamente en el marco conceptual del Cl. Por otra parte, se destaca la presencia de la temática sobre Diversidad e Inclusión dentro de la dimensión de $\mathrm{CH}$, la cual involucra aspectos referidos a la diversidad cultural, igualdad de género y no discriminación.

Con relación al CE, la categoría "Transformación digital" resulta característica de estos tiempos, y aunque las tecnologías de la información y comunicación son mencionadas en el marco conceptual del Cl como un componente del CE, la creciente importancia de estas y su impacto en todos los ámbitos de la empresa, justifican la inclusión de esta categoría como un elemento adicional a considerar dentro del CE. En este sentido, también se destaca en los RI la divulgación de información re- 
ferida a seguridad de la información y privacidad de datos.

Considerando el CR, se enfatiza en la importancia en términos de divulgación de información, de las redes de relaciones de los bancos con la comunidad en la que están insertos. Estas relaciones se materializan en programas de educación financiera, fomento del empleo, de la cultura y del deporte, programas de voluntariado, colaboración con fundaciones, entre otros.

\section{COMENTARIOS FINALES}

El presente trabajo tuvo como objetivo fundamental analizar la divulgación de información sobre Cl en el RI, los efectos de mostrar la complementariedad de la concepción clásica del Cl y sus tres dimensiones con el enfoque del RI, como estrategia de comunicación de información que permite mostrar cómo se desarrolla el proceso de creación de valor a través del tiempo.

Para dar cumplimiento al objetivo planteado se realizó un estudio de tipo exploratorio, que combinó una revisión bibliográfica destinada a mostrar la complementariedad de ambas concepciones a nivel teórico, con la aplicación de un diseño no experimental para la realización de un estudio empírico. Este estudio permitió analizar, en las prácticas de divulgación de información de las empresas, de qué manera se refleja la información referida al Cl a través del RI.

El estudio empírico se realizó sobre las empresas listadas en el mercado de capitales argentino que han publicado su RI, elaborado a partir del marco de referencia del IIRC, en el período 2018-2019, lo que involucra un total de solo tres empresas pertenecientes al sector bancario. Se utilizó una metodología novedosa para el análisis de la información divulgada en los RI: el análisis de datos textuales, el cual se desarrolló en dos etapas. Así, en primer lugar, se aplicó la Clasificación Jerárquica Descendente (CJD), complementada con el análisis de similitud. Y, en la segunda etapa, se realizó el análisis temático.

A partir de la revisión bibliográfica fue posible advertir, desde el punto de vista teórico, una fuerte asociación entre las dimensiones conceptuales del Cl y los componentes de los Capitales Humano, Intelectual y Social y Relacional que se describen en el MIRI. Así el Cl del MIRI, que reúne los inventarios de valor asociados a los intangibles basados en el conocimiento de la organización, se relaciona a la dimensión estructural del Cl. Del mismo modo, es posible identificar que los Capitales Humano y Social y Relacional se encuentran asociados conceptualmente a las dimensiones del Cl que reciben análogas denominaciones.

Con relación al estudio empírico, los resultados obtenidos a partir de los métodos de análisis de datos textuales: CJD y análisis de similitud, permitieron inferir que la información divulgada en los RI responde a la estructura temática del MIRI. Asimismo, fue posible identificar la presencia de los seis capitales, predominando el Industrial, Financiero, Social y Relacional y Humano. Esta evidencia resulta destacable, en tanto los RI analizados pertenecen a empresas del sector bancario. Por otro lado, la CJD y el análisis de similitud, permitieron identificar empíricamente elementos asociados conceptualmente al $\mathrm{Cl}$ y a sus componentes humano, estructural y relacional, lo que resulta consistente con las evidencias que aportan los estudios previos (Ramin, 2013; Melloni, 2015; Beretta y cols., 2019; Terblanche y De Villiers (2019); Salvi y cols., 2020), en los que también se detecta información sobre las tres dimensiones del Cl, en los RI.

El análisis de contenido temático permitió profundizar la indagación con respecto al contenido de la información sobre Cl que las empresas divulgan en los RI, y con la forma en que el Cl es revelado a través de dichos informes. Los resultados de este análisis permitieron identificar categorías temáticas asociadas al $\mathrm{Cl}$, que guardan estrecha relación con los componentes de este. De este modo, se obtuvieron evidencias de la asociación entre la concepción clásica del Cl y el enfoque del RI en las prácticas de divulgación canalizadas a través de dicho informe. Sin embargo, las evidencias también reflejan particularidades en el modo en que el Cl es caracterizado en los RI analizados, que pueden interpretarse según Abhayawansa y cols. (2019), como una adaptación de la contabilidad del Cl al enfoque del RI.

Por lo tanto, sobre la base de estas evidencias, es posible concluir que los RI elabora dos bajo el MIRI tienen potencial para posicionarse como un instrumento apropiado para la divulgación de información sobre Cl y su capacidad de generar valor con relación a otros capitales. Y, en este sentido, el marco del RI reivindicaría la importancia del Cl y de su papel en los procesos de creación de valor de las organizaciones. 
Los hallazgos que derivan de los resultados obtenidos contribuyen a acrecentar la evidencia empírica existente en torno a la divulgación voluntaria de información sobre Cl y, en particular, de aquella que se canaliza a través de los RI, poco estudiados en la literatura previa. Contribuyen también al conocimiento de la potencialidad que posee el RI como estrategia de comunicación del Cl y de su aporte a la creación de valor, que puede ser usada por la gerencia de la empresa para reducir asimetrías informativas y generar confianza en el mercado.

Es preciso señalar también, el aporte de la investigación en cuanto a la metodología usada para el análisis de los RI, porque emplea el análisis de datos textuales, el cual permite combinar técnicas cuantitativas y cualitativas en el estudio de textos no estructurados, complementando al tradicional análisis de contenido, que ha sido de uso habitual en los estudios sobre divulgación de información de $\mathrm{Cl}$ y objeto de diversas críticas. Finalmente, dado que la investigación se contextualizó en el mercado de capitales argentino, sus resultados aportan al conocimiento de las prácticas de revelación de información sobre $\mathrm{Cl}$ a través del RI en el contexto latinoamericano, para el cual los estudios empíricos referidos al RI son muy limitados.

No obstante, la investigación tiene ciertas limitaciones que es necesario dejar plasmadas. Por un lado, el reducido número de RI y de años analizados y, por otro, la pertenencia de las empresas estudiadas a un único sector de actividad. Sin embargo, esta situación no presenta alternativas para su superación, al menos a nivel del mercado de capitales argentino, puesto que, las limitaciones referidas surgen por la escasa cantidad de empresas que elabora este tipo de informes en Argentina (solo tres empresas del sector bancario), dos de las cuales lo hacen recién desde el año 2018. Esta situación se debe al carácter voluntario de presentación del RI en Argentina y al incipiente desarrollo de esta práctica corporativa, que es también común a otros países latinoamericanos.

Por ello, de cara a una futura investigación, resultaría valioso realizar réplicas de este estudio sobre una muestra más amplia, que podría configurarse incluyendo empresas de otros países, preferentemente del entorno latinoamericano. Otra interesante perspectiva, es la relativa a profundizar el estudio empírico ampliando los informes a revisar como fuente de información voluntaria sobre Cl, considerando también a los informes anuales y a 120 las memorias de sostenibilidad. Esto permitiría, además, realizar una comparación entre la información sobre $\mathrm{Cl}$ que las empresas presentan en el RI y la que se divulga a través de otros informes.

\section{REFERENCIAS}

Abhayawansa, S., Aleksanyan, M. y Cuganesan, S. (2018). Conceptualisation of intellectual capital in analysts' narratives: a performative view. Accounting, Auditing \& Accountability Journal, 31(3), 950-969.

Abhayawansa, S., Guthrie, J. y Keynes, M. (2019). Intellectual capital accounting in the age of integrated reporting: a commentary. Journal of Intellectual Capital, 20(1), 2-10.

Al-Hajaya, K., Altarawneh, M. y Altarawneh, B. (2019). Intellectual Capital Disclosure by Listed Companies in Jordan: A Comparative Intersector Analysis. International Review of Management and Marketing, 9(1), 109-116.

Avelar, A., da Silva Oliveira, K., y da Silva Pereira, R. (2019). Education for advancing the implementation of the Sustainable Development Coals: A systematic approach. The International Journal of Management Education, 17(3), 322-337.

Azofra Palenzuela, V., Ochoa Hernández, M., Prieto Moreno, B. y Santidrián Arroyo, A. (2017). Creando valor mediante la aplicación de modelos de capital intelectual. Innovar, 27(65), 25-38.

Bardin, L. (1996). Análisis de contenido. Ediciones Akal.

Benites Lazaro, L., Mello Théry, N., y Lahsen, M. (2017). Business storytelling about energy and climate change: The case of Brazil's ethanol industry. Energy Research \& Social Science, 31, 77-85.

Beretta, V., Demartini, C., y Trucco, S. (2019) Does environmental, social and governance performance influence intellectual capital disclosure tone in integrated reporting? Journal of Intellectual Capital, 20(1), 100-124.

Bollen, L., Vergauwen, P. y Schnieders, S. (2005). Linking intellectual capital and intellectual property to company performance. Management Decision, 43(9), 1161-1185. 
Bontis, N. (1998). Intellectual capital: An exploratory study that develops measures and models. Management Decision, 36(2), 63-76.

Bueno, E., Murcia Rivera, C., Longo, M., Merino, C., Real, H., Fernández, P. y Salmador, M. (2011). Propuesta de nuevo Modelo Intellectus de Medición, gestión e información del Capital Intelectual. CIC. Universidad Autónoma de Madrid.

Bueno, E., Salmador, M. y Merino, C. (2008). Cénesis, concepto y desarrollo del capital intelectual en la economía del conocimiento: Una reflexión sobre el Modelo Intellectus y sus aplicaciones. Estudios de Economía Aplicada, 26(2), 43-63.

Camargo, B. y Justo, A. (2013). IRAMUTEQ: um software gratuito para análise de dados textuais. Temas em psicologia, 21(2), 513-518.

Cañibano, L., Sánchez, M., García, M. y Chaminade, C. (2002). Directrices para la gestión y difusión de información sobre intangibles (Informe de Capital Intelectual). Proyecto Meritum. Fundación Airtel Móvil.

Castilla, F. y Ruiz, C. (2017). La divulgación de intangibles en entidades financieras: un análisis de contenido. Revista Española de Financiación y Contabilidad, 47(1), 81-123.

De Silva, T., Stratford, M. y Clark, M. (2014). Intellectual capital reporting: a longitudinal study of New Zealand companies. Journal of Intellectual Capital, 15(1), 157-172.

De Villiers, C., Rinaldi, L. y Unerman, J. (2014). Integrated Reporting: Insights, gaps and an agenda for future research. Accounting, Auditing \& Accountability Journal, 27(7), 1042-1067.

Dumay, J. (2016). A critical reflection on the future of intellectual capital: from reporting to disclosure. Journal of Intellectual Capital, 17(1), 168-184.

Dumay, J., Bernardi, C., Guthrie, J. y Demartini, P. (2016). Integrated reporting: A structured literature review. Account Forum, 40, 166-185.

Dumay, J. y Cai, L. (2014). A review and critique of content analysis as a methodology for inquiring into IC disclosure. Journal of Intellectual Capital, 15(2), 264-290.
Dumay, J., La Torre, M. y Farneti, F. (2019). Developing trust through stewardship: Implications for intellectual capital, integrated reporting, and the EU Directive 2014/95/EU. Journal of Intellectual Capital, 20, 11-39.

Edvinsson, L. y Malone, M. (1997). Intellectual Capital. Realizing your company's true value by findings its hidden brainpower. Harper Collins Publishers.

Ficco, C. (2020a). Relevancia valorativa de los activos intangibles y del capital intelectual: una revisión de la literatura empírica. Revista Academia \& Negocios, 6(1), 11-30.

Ficco, C. (2020b). Una revisión del concepto de capital intelectual y de las principales alternativas para su identificación y medición. Revista Activos, 18(1), 1-42.

Ficco, C. y Sader, G. (2020). Relevancia valorativa de información financiera y no financiera sobre capital intelectual en empresas cotizadas argentinas. Trabajo presentado en la XXXI Conferencia Académica Permanente de Investigación Contable, La Serena, Chile.

Franzosi, R. (2010). Quantitative Narrative Analysis. SAGE Publications.

Frías-Aceituno, J. V., Rodríguez-Ariza, L. y García-Sánchez, I. M. (2013). The role of the board in the dissemination of integrated corporate social reporting. Corporate Social Responsibility and Environmental Management, 20, 219-233.

Giraldo López, J., Soto Sossa, M. y Mejía Delgado, J. (2018). Características de la información contenida en los reportes integrados: una visión para el mercado integrado de América Latina (MILA). Contaduría Universidad de Antioquia, 73, 163-198.

Coulart, G., Viana, M., y Lucchese-Cheung, T. (2020). Consumer perception towards familiar and innovative foods: the case of a Brazilian product. British Food Journal, 123(1), 125-142.

Hernández, R., Fernández, C. y Baptista, P. (2014). Metodología de la investigación. Mc. Graw Hill.

International Integrated Reporting Council -IIRC(2013). The international IR framework. IIRC. 
Lentjušenkova, O. y Lapina, I. (2016). The transformation of the organization's intellectual capital: from resource to capital. Journal of Intellectual Capital, 17(4), 610-631.

Macias, H. y Farfan Lievano, A. (2017). Integrated reporting as a strategy for firm growth: multiple case study in Colombia. Meditari Accountancy Research, 25(4), 605-628.

Melloni, G. (2015). Intellectual capital disclosure in integrated reporting: an impression management analysis. Journal of Intellectual Capital, 16(3), $661-680$.

Nahapiet, J. y Ghoshal, S. (1998). Social capital, intellectual capital, and the organizational advantage. The Academy of Management Review, 23(2), 242-266.

Navarrete, O. J. y Gallizo, L. J. (2018). Reporte Integrado como herramienta de transparencia empresarial. Revista Academia \& Negocios, 4(2), 71-82.

Ochoa Hernández, M., Prieto Moreno, B. y Santidrián Arroyo, A. (2010). Estado actual de los modelos de capital intelectual y su impacto en la creación de valor en empresas de Castilla y León. Revista de Investigación Económica y Social de Castilla y León, 13, 15-205.

Ramin, K. (2013). Integrated Reporting and Intellectual Capital: Concepts and Possible Solutions. In Fischer, T. \& Wulf, I. (Eds.), Wissensbilanzen im Mittelstand (pp. 109-124) Schaeffer-Poeschel.

Reinert, A. (1983). Une méthode de classification descendante hiérarchique: application à l'analyse lexicale par contexte. Les cahiers de l'analyse des données, 8(2), 187-198.

Rivera Arrubla, Y., Zorio Grima, A. y García Benau, M. (2016). El concepto de informe integrado como innovación en reporting corporativo. Journal of Innovation \& Knowledge, 1(3), 144155.
Roos, G., Roos, J., Dragonetti, N. y Edvinsson, L. (2001). Capital Intelectual. Paidós Empresa.

Salvi, A., Vitolla, F., Giakoumelou, A., Raimo, N., y Rubino, M. (2020). Intellectual capital disclosure in integrated reports: the effect on firm value. Technological Forecasting and Social Change, 160, 2020, 228-235.

Sánchez, D., Rivera, J. y Brand, R. (compiladores) (2019). La investigación e información corporativa desde la perspectiva de los reportes integrados y el Gobierno corporativo. Fondo Editorial Universitario Servando Garcés de la Universidad Politécnica Territorial de Falcón Alonso Gamero (UPTAG).

Sihotang, P. y Sanjaya, Y. (2014). Reporting intellectual capital in annual reports: Evidence from Indonesia. Indonesian Capital Market Review, 1(2), 125-152.

Stewart, T. (1998). La nueva riqueza de las organizaciones: El capital intelectual. Ediciones Granica S.A.

Tejedo, F. (2016). La información de los recursos intangibles ocultos: ¿memorias de sostenibilidad o informe anual? European Research on Management and Business Economics, 22(2), 101-109.

Terblanche, W. y De Villiers, C. (2019). The influence of integrated reporting and internationalization on intellectual capital disclosures. Journal of Intellectual Capital, 20(1), 40-59.

Vaara, E. (2010). Taking the linguistic turn seriousIy: Strategy as A multifaceted and interdiscursive phenomenon. In J. Baum \& J. Lampel (Eds.), The Globalization of Strategy Research (Advances in Strategic Management, Vol. 27, pp. 29-50). Emerald Group Publishing.

Wild, S. y van Staden, C. (2013). Integrated reporting: initial analysis of early reporters - an institutional theory approach. Paper presented at the 7th Seventh Asia Pacific Interdisciplinary Research in Accounting Conference, Kobe, Japan. 